\title{
Essential Roles of Sall Family Genes in Kidney Development
}

\author{
Ryuichi NISHINAKAMURA and Kenji OSAFUNE \\ Division of Integrative Cell Biology, Institute of Molecular Embryology and Genetics, Kumamoto University, \\ Kumamoto, 860-0811, Japan
}

\begin{abstract}
We isolated a mouse Sall1, a mammalian homologue of the Drosophila region-specific homeotic gene spalt (sal), and found that mice deficient in Sall1 die in the perinatal period from kidney agenesis. Sall1 is expressed in the metanephric mesenchyme surrounding the ureteric bud, and the homozygous deletion of Sall1 results in an incomplete ureteric bud outgrowth. Therefore Sall1 is essential for ureteric bud invasion, the initial key step for metanephros development. We also set up an in vitro culture system, using NIH3T3 cells stably expressing Wnt4 as a feeder layer, to identify kidney progenitors in the metanephric
\end{abstract}

mesenchyme. In this culture condition, a single renal progenitor in the mesenchyme forms colonies consisting of several types of epithelial cells that exist in glomeruli and renal tubules. We found that only cells strongly expressing Sall1 (Sall1-GFPhigh cells) form colonies and that they reconstitute a three-dimensional kidney structure in an organ culture setting. Thus our colony-forming assay, which identifies multipotent progenitors in the embryonic mouse kidney, can be used for examining mechanisms of renal progenitor differentiation.

Key words: kidney, development, Sall1, metanephric mesenchyme, progenitor.

\section{Three kidneys during development}

The kidney function is impaired in a variety of disease states, but all existing treatments for kidney diseases are conservative, and there is essentially no therapy available for curing renal function per se. Regenerating kidney should be tough, but the hints are likely to lie in kidney development. We wish to challenge this difficult project to reveal the mechanisms of kidney development and would like to link the information to strategies for regenerating kidney.

The kidney develops in three stages: pronephros, mesonephros, and metanephros. The nephric duct (Wolffian duct) develops in the craniocaudal direction from the intermediate mesoderm and acts on the surrounding mesenchyme as an inducer of epithelial transformation to nephric tubules. The pronephric and mesonephric tubules and the anterior portion of the Wolffian duct eventually degenerate, and in mammals the metanephros becomes the permanent kidney. Although the pronephros represents a true excretory organ in fish and amphibians, it remains a rudimentary and transient structure in mouse.

\section{Using frog embryos to identify Sall genes}

The animal cap is a tiny portion of the presumptive ectoderm of Xenopus embryos in the blastula stage. In the presence of activin, animal caps differentiate into a variety of tissues. A combination of activin plus retinoic acid induces pronephric tubules efficiently and selectively [1]. We used this animal cap system to identify molecules ex- pressed in pronephros and potentially in mesonephros and metanephros. Thousands of animal caps treated with activin plus retinoic acid were collected at various time points and subjected to a variety of subtraction procedures (Fig. 1). One of the obtained molecules was Xsal-3, which is homologous to the Drosophila region-specific homeotic gene spalt ( $\mathrm{sal}$ ) and has multiple double-zinc finger motifs that are characteristic of the sal gene family [2]. We also isolated a mouse homologue (Sall1) and found it to be expressed in otic vesicles, limb buds, anus, hearts, and kidneys (metanephric mesenchyme) [3].

Drosophila sal is the region-specific homeotic gene characterized by unique multiple double-zinc finger motifs. First identified by virtue of its capacity to promote terminal differentiation, sal is expressed in anterior and posterior compartments of Drosophila, and mutations in sal cause head and tail segments to develop trunk structures [4]. It also plays a critical role in wing development and is expressed at the anterior/posterior boundary of wing imaginal discs. Its expression is controlled by $d p p$ (BMP-4 orthologue), the expression of which is highest at the boundary, which in turn is controlled by hedgehog, expressed in the posterior compartment [5]. An overexpression of $d p p$ broadens the expression domains of $s a l$; thus $s a l$ may be a downstream target of $d p p$. It was also found to be downstream of the wingless signal in the Drosophila tracheal system, and sal deletion results in an absence of dorsal trunks of the trachea [6].

Received on Nov 22, 2005; accepted on Apr 19, 2006; released online on Apr 22, 2006; doi:10.2170/physiolsci.M95 Correspondence should be addressed to: Ryuichi Nishinakamura, Division of Integrative Cell Biology, Institute of Molecular Embryology and Genetics, Kumamoto University, 2-2-1 Honjo, Kumamoto, 860-0811, Japan Phone: +81-96-373-6615, Fax: +81-96373-6618, E-mail: ryuichi@kaiju.medic.kumamoto-u.ac.jp 


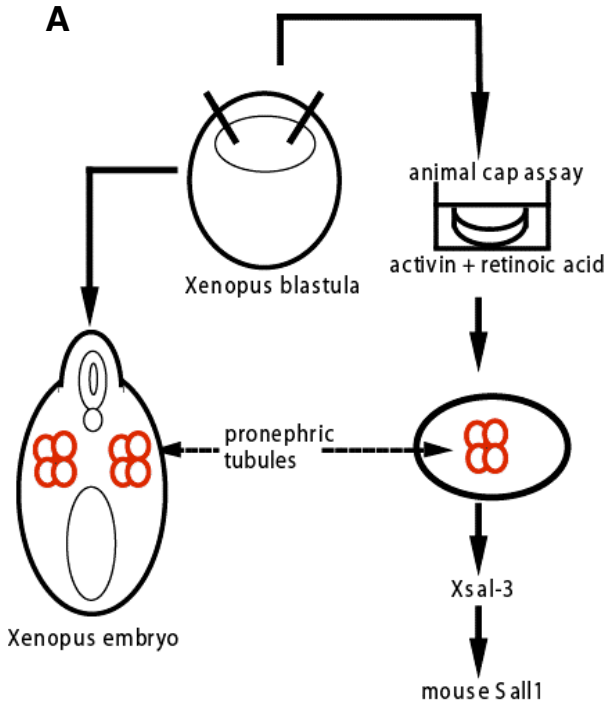

B

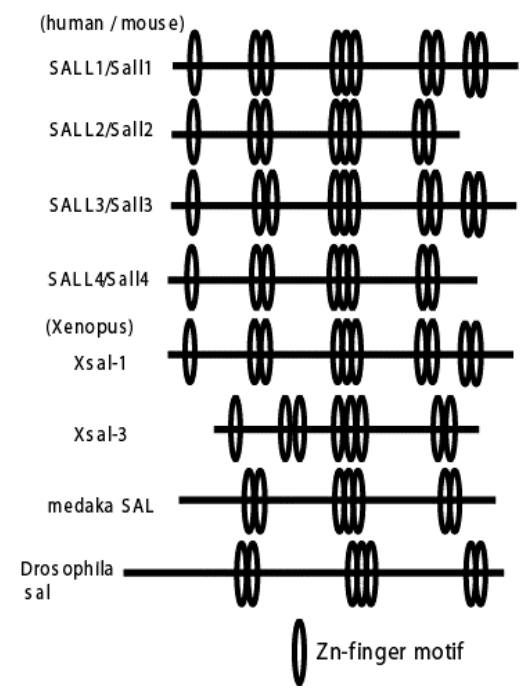

Fig. 1. Pronephros formation in animal caps in Xenopus laevis and cloning of Sall genes. A: Pronephros formation in animal caps treated with activin plus retinoic acid. Animal caps treated for three hours contain pronephric tubules 3 days after the treatment. B: Sall family genes conserved from Drosophila to humans. Ovals indicate zinc-finger motifs.

\section{Sall1 is essential to kidney development}

In metanephros development, the metanephric mesenchyme induces a sprouting of the ureteric bud from the caudal region of the Wolffian duct (Fig. 2). Signals from the mesenchyme cause a further branching of the ureteric bud, thus forming the collecting ducts and the ureters. Reciprocally, the ureteric bud invades the mesenchyme and induces epithelialization and differentiation of the mesenchyme into the glomeruli, the loop of Henle, and the proximal and distal tubules.

When we generated Sall1 knockout mice, all of the homozygous mice died within 24 hours after birth, and kidney agenesis or severe dysgenesis was present (Fig. 3) [3]. About a third had no kidneys or ureters bilaterally (Fig. 3B). The remaining mice had either unilateral kidney agenesis or bilateral hypolasia (Fig. 3C). At day 11.5 of gestation (E11.5), the ureteric bud invades the metanephric mesenchyme, and subsequent reciprocal interaction between these two tissues leads to a development of a metanephric kidney (Fig. 3D). In Sall1-null mice, morphologically distinct metanephric mesenchyme was formed, though the size was reduced (Fig. 3E). In contrast, the ureteric bud formed, but failed to invade the metanephric mesenchyme. Thus a loss of Sall1 leads to a failure of ureteric bud invasion into the mesenchyme, the initial key step for metanephros development. The development of other organs, including brain, adrenal glands, bladder, testis, and ovary, was normal.

\section{Molecular mechanisms of kidney development}

The molecular mechanisms of kidney development have been revealed mostly by gene targeting. Here I briefly introduce some of the findings (Fig. 4), but more-detailed reviews are also available $[7,8]$. A member of the TGF- $\beta$ superfamily, GDNF (glial cell-line-derived neurotrophic factor), expressed in the mesenchyme, acts on a

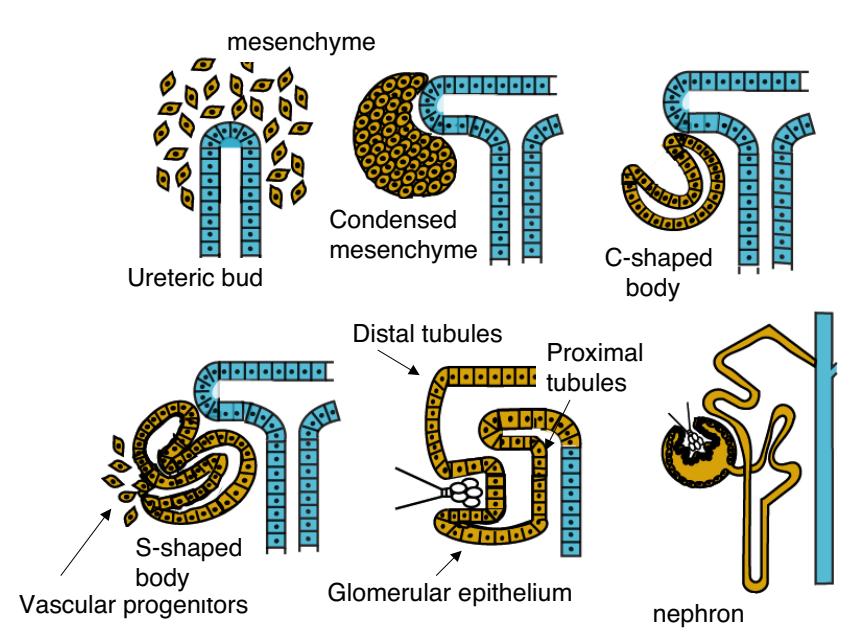

Fig. 2. Metanephros development. See details in the text. The mesenchyme gives rise to the glomeruli, proximal and distal tubules, and the loops of Henle, but the ureteric bud differentiates into the collecting ducts and the ureters. Sall1 is expressed in the mesenchyme and is essential for the ureteric bud attraction to the mesenchyme.

receptor tyrosine kinase Ret distributed in the ureteric bud epithelium and induces a branching of the ureter. Thus the null mutants of GDNF and Ret show perturbation of ureter invasion. The transcription factors Six1, Pax2, and Eya1 also control kidney development, partly by upregulating GDNF expression. In contrast, secreted proteins Slit 2 and BMP4 antagonize the GDNF action and inhibit the ectopic formation of ureteric buds.

Reciprocal signals from the ureteric bud to the mesenchyme remained unidentified for a long period. Recently, Wnt9b was reported to be a ureter-derived regulator for mesenchymal to epithelial conversion, and mice that are deficient in Wnt9b lack the initial induction of the mesenchyme, including Wnt4 expression [9]. Mice lacking Wnt-4 fail to form pretubular aggregates, a transitional 

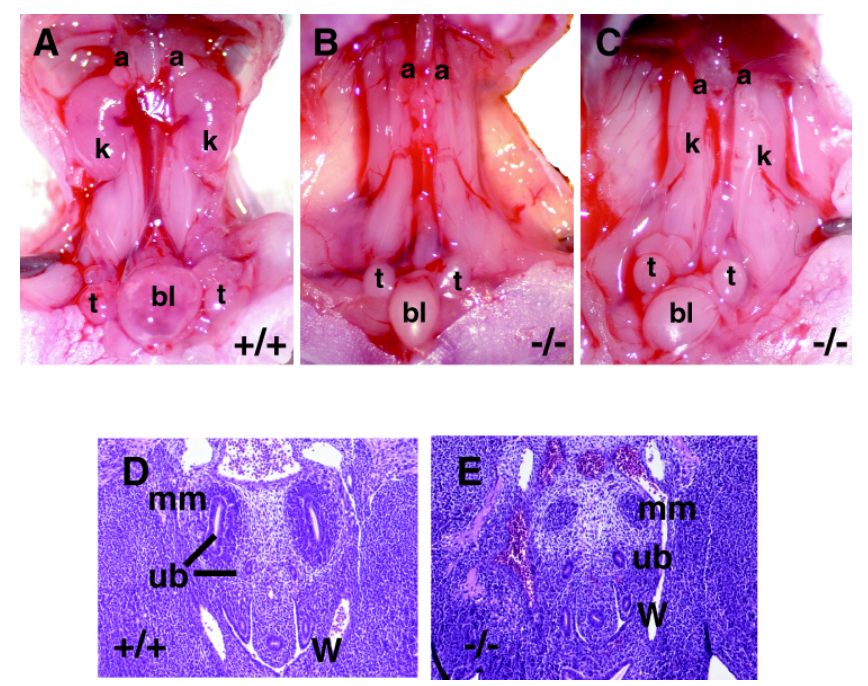

Fig. 3. Kidney phenotypes in Sall1-deficient mice. A: Kidneys (k) of wild-type newborn. Urinary bladder (bl) is filled with urine. B: Kidneys of Sall1-deficient newborn. Note that kidneys are absent and the urinary bladder is not inflated with urine. Other organs, such as adrenal glands (a) and testes $(\mathrm{t})$, are normal. C: Kidneys of another Sall1-deficient newborn with severe bilateral kidney hypoplasia. Urine is absent in the bladder. $\mathbf{D}$ : Metanephros in wild-type mice at $11.5 \mathrm{dpc}$. The ureteric bud (ub) branches from the Wolffian duct (W) and metanephric mesenchymes $(\mathrm{mm})$ are condensed around the bulging ureteric bud. E: Metanephros in Sall1-deficient mice at $11.5 \mathrm{dpc}$. The metanephric mesenchyme is formed, but it is reduced in size and is not invaded by the ureteric bud.

state from mesenchyme to tubules; thus Wnt4 functions downstream of Wnt9b and acts as an autoinducer of the mesenchyme to epithelial transition.

\section{Potential mechanisms related to the Sall family}

Several possibilities can explain the Sall1 knockout phenotype. Soluble factors secreted from the mesenchyme to attract the ureteric bud may be impaired in the absence of Sall1. GDNF is a potential candidate, and indeed the most severe cases in Sall1 knockout were similar to those seen in $G D N F$-deficient mice. GDNF is ex- pressed in the metanephric mesenchyme and is a key molecule for attracting the ureteric bud, acting through the Ret receptor. $G D N F$ expression in the mesenchyme is reduced in Sall1 knockout at E11.5, but not at E10.5 (before ureteric bud invasion). Therefore Sall1 is not absolutely required for GDNF expression. It is, however, still possible that reduced levels of GDNF may explain the ureteric bud phenotype of Salll knockout.

It was reported that Sall1 also functions as a transcriptional repressor by localizing in the heterochromatin and interacting with the components of chromatin remodeling complexes, such as histone deacetylase (HDAC)1, HDAC2, retinoblastoma-associated protein 46/48 (RbAp46/48), metastasis-associated protein (MTA)1, and MTA2 [10,11]. Sall1 may bind to heterochromatin directly and recruit molecules that form heterochromatin complexes. If this hypothesis is valid, Sall1 may repress inhibitors, thereby activating kidney development. Direct targets that are repressed by Sall1 remain unknown, and the identification of such targets is needed to fully explain kidney phenotypes of Sall1-deficient mice. Sall1 contains 10 zinc finger motifs, most of which are clustered in duplex or triplet, but it is not known which zinc finger domain is involved in DNA binding or in transactivation. A search for Sall1 target genes is under way in various laboratories.

\section{Kidney abnormalities caused by human SALL1 mutations}

Humans and mice have four known sal-related genes, respectively (SALL1-4 for humans and Sall1-4 for mice). Mutations in SALL1 have been associated with TownesBrocks syndrome, an autosomal dominant disease with features of dysplastic ears, preaxial polydactyly, imperforate anus, and, less commonly, kidney and heart anomalies [12]. Mice deficient in Sall1 show kidney agenesis or severe dysgenesis, but other phenotypes observed in human disease are not apparent, as described above [3]. This discrepancy could be explained by truncated SALL1 proteins resulting from human mutations, possibly functioning in a dominant-negative manner, because mutant mice
A

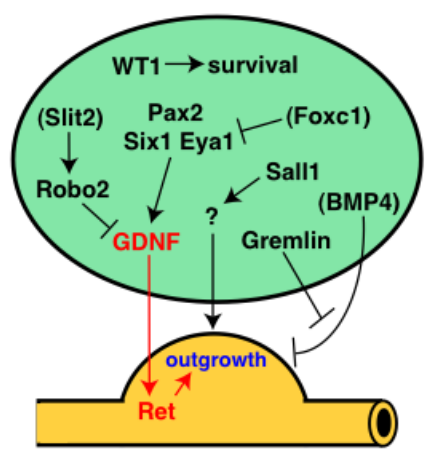

B

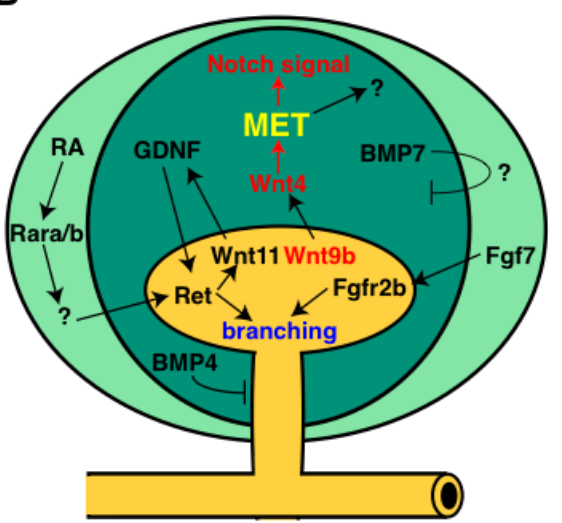

Fig. 4. Molecules involved in kidney development. A: Mesenchyme secretes GDNF and attracts the ureterid bud. B: Wnt9b secreted from the ureteric bud upregulates Wnt4 in the mesenchyme. Wnt4 in turn plays a role in mesenchymal-to-epithelial transition (MET). 
A
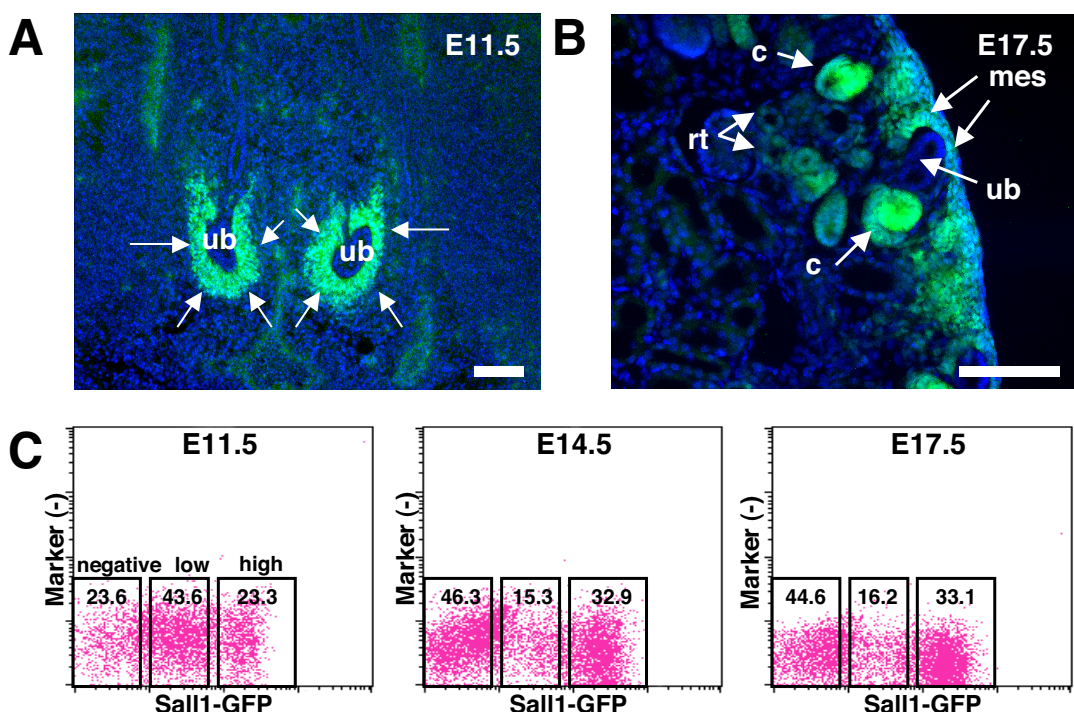

E14.5
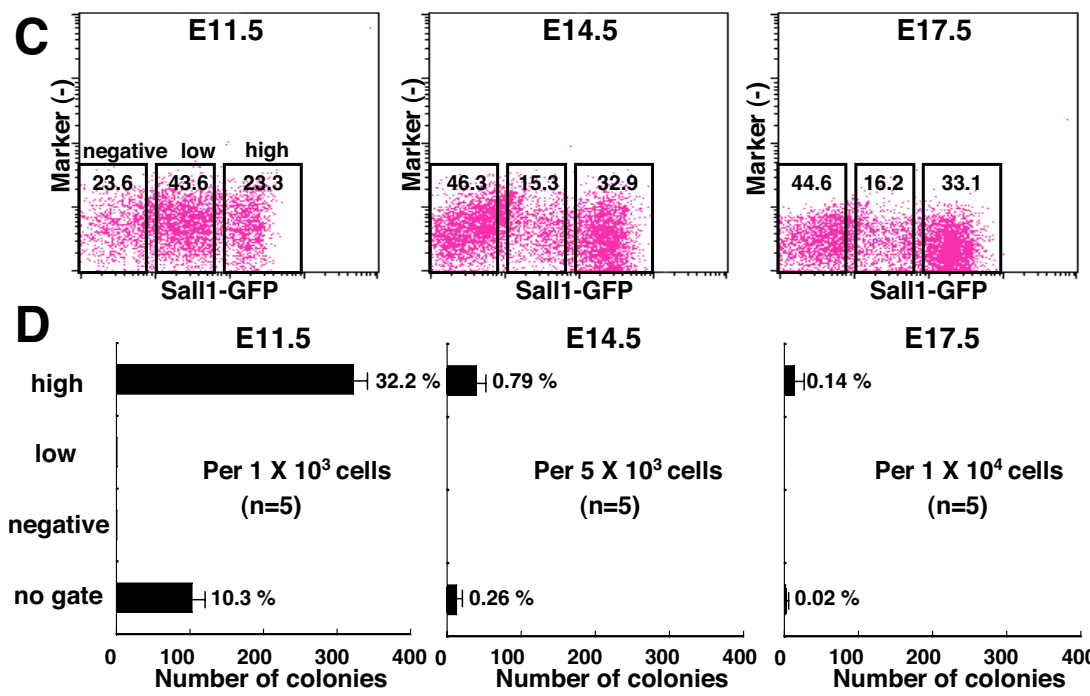

E17.5

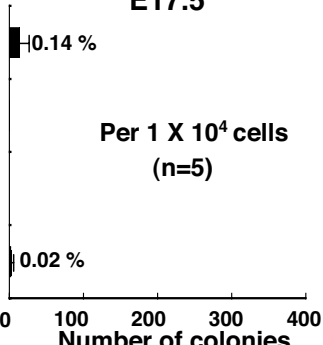

Fig. 5. Colony-forming progenitors exist in the Sall1-GFP high subpopulation of the metanephros. A, B: Cryosections of the metanephros of Sall1-GFP knock-in mouse (A: E11.5, B: E17.5). ub: ureteric bud; mes: mesenchyme; C: C-shaped body; rt: renal tubule; blue: DAPI. Scale bars: $50 \mu \mathrm{m}$. C. Metanephros contains three subpopulations (Sall1-GFPhigh, Sall1-GFPlow, and Sall1-GFPnegative). The percentages of the subpopulations at each fetal stage are shown. D. Numbers of colonies in each subpopulation derived from E11.5 mesenchyme, E14.5, and E17.5 metanephros. The number of colonies were counted after a 20-day culture.

that produce a truncated Sall1 protein exhibit more severe defects than Sall1-null mice, including renal agenesis, exencephaly, and limb and anal deformities [13]. Sall2-deficient mice show no apparent phenotypes, and mice lacking both Sall1 and Sall2 show kidney phenotypes comparable to those of Sall1 knockout [14]. Sall3-null mice die on the first postnatal day, and deficiencies in cranial nerves and abnormalities in the oral structures are present [15]. Mutations of SALL4 cause an autosomal dominant disorder, Okihiro syndrome, characterized by limb deformity and eye movement deficits and, though less common, anorectal and kidney anomalies [16, 17], and we are currently generating Sall4-deficient mice. A generation of mice lacking all Sall genes would be necessary to address the developmental roles of the Sall family.

\section{Identification of kidney mesenchymal genes by a combination of microarray analysis and Sall1-GFP knock-in mice}

Gene targeting has revealed several genes essential to kidney development, yet more genes are probably involved in the generation of a complicated kidney. In the embryonic kidney, Sall1 is expressed abundantly in mesenchyme-derived structures from condensed mesenchyme and S- and comma-shaped bodies to renal tubules and podocytes. We generated mice in which a green fluorescent protein $(G F P)$ gene was inserted into the Sall1 locus, and we isolated the $G F P$-positive population from embryonic kidneys of these mice by fluorescein-activated cell sorting (FACS) [18]. The GFP-positive population indeed expressed mesenchymal genes, but the negative population expressed genes in the ureteric bud. To systematically search for genes expressed in the mesenchymederived cells, we compared gene expression profiles in the GFP-positive and GFP-negative populations by using microarray analysis followed by in situ hybridization. We detected many genes known to be important for metanephros development, including Sall1, GDNF, Raldh2, Pax 8 , and FoxD1, and genes expressed abundantly in the metanephric mesenchyme, such as Unc4.1, Six2, Osr-2, and $P D G F C$. We also found groups of genes, including $S S B-4$, Smarcd3, $\mu$-Crystallin, and TRB-2, that are not known to be expressed in the metanephric mesenchyme. Therefore a combination of microarray technology and Sall1-GFP mice is useful for a systematic identification of genes expressed in the developing kidney. Finding essential genes from this large list requires efficient and rapid screening. siRNA technology has recently become one potent method, but generating the knockout mice of each candidate gene is necessary for proof.

\section{Identification of multipotent progenitors in the embryonic mouse kidney by a novel colony- forming assay}

Eventually we hope to reconstruct the kidney by utilizing available knowledge on development. The derivation 


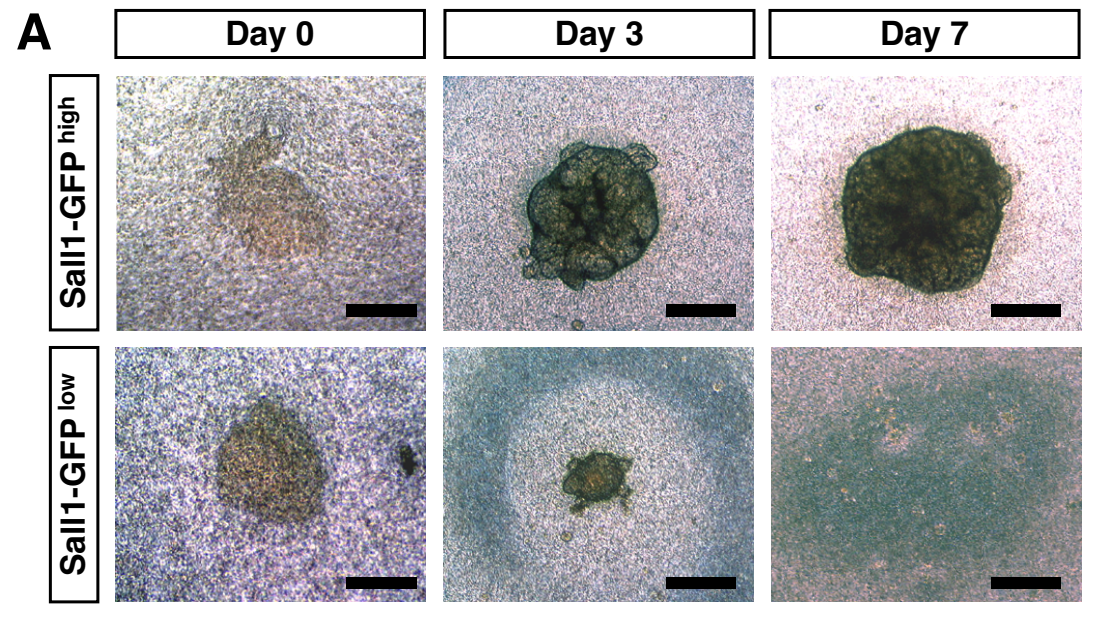

Fig. 6. Sall1-GFPhigh mesenchyme differentiates into renal epithelia in organ culture. A: Three subpopulations in E11.5 mesenchyme (Sall1-GFPhigh, Sall1-GFPlow, and Sall1-GFPnegative) were cultured on 3T3Wnt4 feeder cells in an organ culture setting. Only Sall1-GFPhigh cells (upper panels) differentiated into the kidney structure; the Sall1-GFPlow cells (lower) disappeared. B: Hematoxylin-eosin staining of sections of Sall1-GFPhigh aggregates at day 10 . Tubule$(\mathrm{t})$ and glomerulus-like structures $(\mathrm{g})$ are seen. C: Double staining with WT1 (red, podocyte marker) and LTL (green, proximal tubule marker) of Sall1-GFPhigh aggregates. Scale bars: (A) $500 \mu \mathrm{m}$; (B, C) $25 \mu \mathrm{m}$.

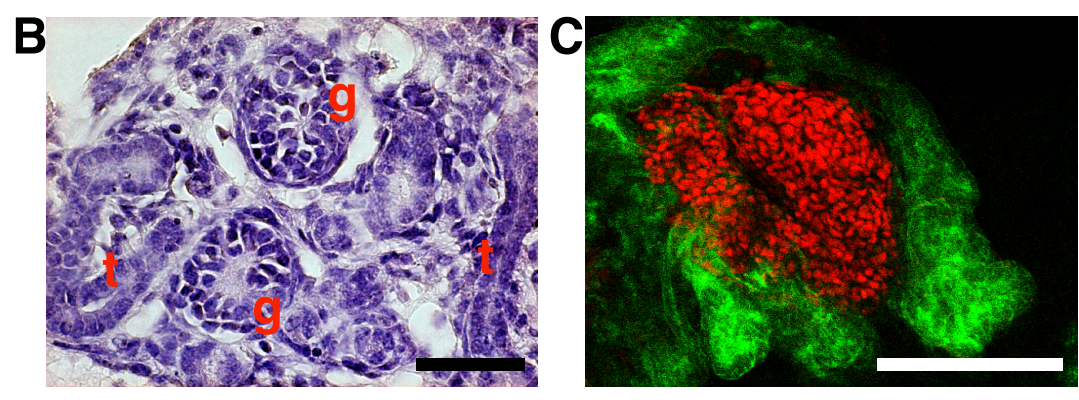

of kidney cells from a variety of sources such as embryonic stem cells and bone marrow would be a challenge. For this purpose, assay systems that recognize the existence and frequency of kidney progenitors, both in vitro and in vivo, are needed. We established a novel colony-forming assay system by using $3 \mathrm{~T} 3$ cells expressing Wnt4 (3T3Wnt4) to identify renal progenitors in the metanephric mesenchyme [19].

We cultured dissociated cells from the metanephric mesenchyme of E11.5 embryos, using 3T3Wnt4 as a feeder layer in a serum-free condition. This culture condition resulted in a formation of sheetlike colonies, and these colonies contain differentiated cells expressing marker genes for epithelia in glomeruli (podocyte), proximal or distal tubules, and the loop of Henle. We also confirmed by clone sorting that these colonies are derived from a single progenitor with multipotent differentiating capacity.

We next attempted to prospectively identify the renal progenitor cells by using Sall1-GFP knock-in mice [18]. Because Sall1 is expressed in mesenchyme-derived tissues, GFP was detected in the mesenchyme around the ureteric bud at E11.5 in the Sall1-GFP heterozygous mouse (Fig. 5A, arrows). At E17.5, GFP-expressing cells were observed in the mesenchyme near the surface, as well as in C- or S-shaped bodies and parts of renal tubules (Fig. 5B). By flowcytometrical analysis, three subpopulations were fractionated based on the expression of Sall1GFP: Sall1-GFPhigh, Sall1-GFPlow, and Sall1-GFPnegative (Fig. 5C), and the numbers of the colony-forming progen- itors in each subpopulation were examined by the use of the low-density culture on 3T3Wnt4 (Fig. 5D). At E11.5, colonies were formed exclusively from Sall1-GFPhigh population, and not from the Sall1-GFPlow or Sall1GFPnegative population. At E14.5 and E17.5, colonies were also formed only from Sall1-GFPhigh subpopulations, but the frequency of colony-forming progenitors decreased as gestation proceeded. These results indicate that renal progenitors with multipotent differentiating capacity are included in cell populations strongly expressing Sall1 throughout gestation periods.

\section{Sall1-GFPhigh mesenchyme reconstitutes a three- dimensional structure in organ culture}

We examined the in vitro differentiation capacity of three subpopulations in E11.5 mesenchyme by modifying the organ culture of mesenchyme rudiments [19]. Sall1GFPhigh, Sall1-GFPlow, and Sall1-GFPnegative cells were separated by flowcytometry, aggregated to form a cell pellet by centrifugation and cultured on 3T3Wnt4 feeder cells in an organ culture setting. Starting from day 3 in culture, tubulogenesis was observed only in the aggregate of Sall1-GFP high population (Fig. 6A, upper panels), but that from Sall1-GFP low or Sall1-GFP negative did not differentiate and disappeared by day 7 (Fig. 6A, lower panels; data of Sall1-GFPnegative not shown). In sections of the Sall1-GFPhigh aggregate (Fig. 6B), many tubule- (t) and glomerulus-like structures $(\mathrm{g})$ were observed, and the expression of markers for glomerular podocyte (WT1, Fig. 


\section{R. NISHINAKAMURA and K. OSAFUNE}

6C, red) and proximal tubule (LTL, green) was confirmed by confocal microscopy. These data suggest that only Sall1-GFPhigh cells differentiate into renal epithelia in vitro in a three-dimensional setting, besides forming colonies. Thus our colony-forming assay, which identifies multipotent progenitors in the embryonic mouse kidney, can be used for examining the mechanisms of renal progenitor differentiation as well as for the derivation of kidney progenitors from a variety of cell sources.

We thank Minoru Takasato for the initial characterization of Sall1-GFP mice and Andreas Kispert for providing 3T3Wnt4 cells.

\section{REFERENCES}

1. Uochi T, Asashima M. Sequential gene expression during pronephric tubule formation in vitro in Xenopus ectoderm. Dev Growth Differ. 1996;38:625-35.

2. Onuma Y, Nishinakamura R, Takahashi S, Yokota T, Asashima M. Molecular cloning of a novel Xenopus spalt gene (Xsal-3). Biochem Biophys Res Commun. 1999;264:151-6.

3. Nishinakamura R, Matsumoto Y, Nakao K, Nakamura K, Sato A, Copeland NG, Gilbert DJ, Jenkins NA, Scully S, Lacey DL, Katsuki M, Asashima M, Yokota T: Murine homolog of SALL1 is essential for ureteric bud invasion in kidney development. Development. 2001;128:3105-15.

4. Kuhnlein RP, Frommer G, Friedrich M, Gonzalez-Gaitan M, Weber A, WagnerBernholz JF, Gehring WJ, Jackle H, Schuh R. spalt encodes an evolutionarily conserved zinc finger protein of novel structure which provides homeotic gene function in the head and tail region of the Drosophila embryo. EMBO J. 1994;13:168-79.

5. de Celis JF, Barrio R, Kafatos FC. A gene complex acting downstream of dpp in Drosophila wing morphogenesis. Nature. 1996;381:421-4.

6. Chihara T, Hayashi S. Control of tracheal tubulogenesis by Wingless signaling. Development. 2000;127:4433-4442.

7. Vainio $S$, Lin Y. Coordinating early kidney development: lessons from gene targeting. Nat Rev Genet. 2002;3:533-43.
8. Perantoni AO. Renal development: perspectives on a Wnt-dependent process. Semin Cell Dev Biol. 2003;14:201-8.

9. Carroll TJ, Park JS, Hayashi S, Majumdar A, McMahon AP. Wnt9b plays a central role in the regulation of mesenchymal to epithelial transitions underlying organogenesis of the mammalian urogenital system. Dev Cell. 2005;9:283-92.

10. Netzer C, Rieger L, Brero A, Zhang CD, Hinzke M, Kohlhase J, Bohlander SK. SALL1, the gene mutated in Townes-Brocks syndrome, encodes a transcriptional repressor which interacts with TRF1/PIN2 and localizes to pericentromeric heterochromatin. Hum Mol Genet. 2001;10:3017-24.

11. Kiefer SM, McDill BW, Yang J, Rauchman M. Murine Sall1 represses transcription by recruiting a histone deacetylase complex. J Biol Chem. 2002;277:14869-76.

12. Kohlhase J, Wischermann A, Reichenbach $\mathrm{H}$, Froster $\mathrm{U}$, Engel W. Mutations in the SALL1 putative transcription factor gene cause Townes-Brocks syndrome. Nat Genet. 1998:18:81-3.

13. Kiefer SM, Ohlemiller KK, Yang J, McDill BW, Kohlhase J, Rauchman M. Expression of a truncated Sall1 transcriptional repressor is responsible for Townes-Brocks syndrome birth defects. Hum Mol Genet. 2003;12:2221-7.

14. Sato A, Matsumoto Y, Koide U, Kataoka Y, Yoshida N, Yokota T, Asashima M, Nishinakamura R. Zinc finger protein Sall2 is not essential for embryonic and kidney development. Mol Cell Biol. 2003;23:62-9.

15. Parrish M, Ott T, Lance-Jones C, Schuetz G, Schwaeger-Nickolenko A, Monaghan AP. Loss of the Sall/3 gene leads to palate deficiency, abnormalities in cranial nerves, and perinatal lethality. Mol Cell Biol. 2004;24:7102-12.

16. Al-Baradie R, Yamada K, St Hilaire C, Chan WM, Andrews C, McIntosh N, Nakano M, Martonyi EJ, Raymond WR, Okumura S, Okihiro MM, Engle EC. Duane radial ray syndrome (Okihiro syndrome) maps to $20 \mathrm{q} 13$ and results from mutations in SALL4, a new member of the SAL family. Am J Hum Genet. 2002;71:1195-9.

17. Kohlhase J, Heinrich M, Schubert L, Liebers M, Kispert A, Laccone F, Turnpenny $\mathrm{P}$, Winter RM, Reardon W. Okihiro syndrome is caused by SALL4 mutations. Hum Mol Genet. 2002;11:2979-87.

18. Takasato M, Osafune K, Matsumoto Y, Kataoka Y, Yoshida N, Meguro H, Aburatani $\mathrm{H}$, Asashima M, Nishinakamura R. Identification of kidney mesenchymal genes by a combination of microarray analysis and Sall1-GFP knockin mice. Mech Dev. 2004;121:547-57.

19. Osafune K, Takasato M, Kispert A, Asashima M, Nishinakamura R. Identification of multipotent progenitors in the embryonic mouse kidney by a novel colonyforming assay. Development. 2006;133:151-61. 